\title{
Differential response of human and rat epoxide hydrolase to polycyclic aromatic hydrocarbon exposure; studies using precision- cut tissue slices
}

\author{
Daphnee S Pushparajah, Meera Umachandran, Kathryn E Plant, Nick Plant and \\ Costas Ioannides \\ Molecular Toxicology Group, School of Biomedical and Molecular Sciences, \\ University of Surrey, Guildford, Surrey, GU2 7XH, UK
}

Key words: epoxide hydrolase; polycyclic aromatic hydrocarbons; precision-cut slices; tissue differences; species differences; chemical carcinogenesis.

Corresponding author: Professor C Ioannides

Molecular Toxicology Group

School of Biomedical and Molecular Sciences

University of Surrey

Guildford, Surrey

GU2 7XH, UK

Tel. No: +44 1483689709

Fax No: +44 1483686401

E-mail: c.ioannides@surrey.ac.uk 


\section{Abstract}

The potential of polycyclic aromatic hydrocarbons (PAHs) to modulate microsomal epoxide hydrolase activity, determined using benzo[a]pyrene 5-oxide as substrate, in human liver, was evaluated and compared to rat liver. Precision-cut liver slices prepared from fresh human liver were incubated with six structurally-diverse PAHs, at a range of concentrations, for 24 hours. Of the six PAHs studied, benzo[a]pyrene, dibenzo[a,h]anthracene and fluoranthene gave rise to a statistically significant increase in epoxide hydrolase activity, which was accompanied by a concomitant increase in epoxide hydrolase protein levels determined by immunoblotting. The other PAHs studied, namely dibenzo[a,1]pyrene, benzo[b]fluoranthene and 1methylphenanthrene, influenced neither activity nor enzyme protein levels. When rat slices were incubated under identical conditions, only benzo[a]pyrene and dibenzo[a,h]anthracene elevated epoxide hydrolase activity, which was, once again accompanied by a rise in protein levels. At the mRNA level, however, all six PAHs caused an increase, albeit to different extent. In rat, epoxide hydroxylase activity in lung slices was much lower than in liver slices. In lung slices, epoxide hydrolase activity was elevated following exposure to benzo[a]pyrene and dibenzo[a,1]pyrene and, to a lesser extent, 1-methylphenanthrene; similar observations were made at the protein level. At both activity and protein levels extent of induction was far more pronounced in the lung compared with the liver. It is concluded that epoxide hydrolase activity is an inducible enzyme by PAHs, in both human and rat liver, but induction potential by individual PAHs varies enormously, depending on the nature of the compound involved. Marked tissue differences in the nature of PAHs stimulating activity in rat lung and liver were noted. Although in the rat basal lung epoxide hydrolase activity is much lower than liver, it is more markedly inducible by PAHs. 


\section{Introduction}

The toxicity and carcinogenicity of many chemicals, both naturally-occurring and anthropogenic, are inextricably linked to their metabolism to reactive, electrophilic intermediates that interact covalently with vital cellular macromolecules leading to mutations and toxicity. Such metabolites include epoxides, which have been implicated in the toxicity/carcinogenicity of many structurally-diverse chemicals including polycyclic aromatic hydrocarbons [1], mycotoxins such as aflatoxin $\mathrm{B}_{1}[2]$, and halogenated aliphatic compounds such as vinyl chloride [3]; their formation is most frequently catalysed by cytochromes $\mathrm{P} 450$.

The principal protective mechanism of the living organism is to deactivate the epoxides by metabolically converting them to inactive, readily excretable products. The two most important enzyme systems catalysing the detoxication of epoxides are the microsomal epoxide hydrolase [4] and the cytosolic glutathione $S$-transferases [5]. The former inserts water to the epoxide to yield a dihydrodiol, and the latter catalyses the conjugation of epoxides with reduced glutathione; the glutathione conjugate is usually further metabolically processed and is excreted as the mercapturate. In the case of the primary epoxides of polycyclic aromatic hydrocarbons (PAHs), however, microsomal epoxide hydrolase converts primary epoxides to the corresponding transdihydrodiols, which are the precursors for the generation of dihydrodiol epoxides, following cytochrome P450-mediated oxidation, the ultimate carcinogenic species; these epoxides do not serve as substrates of epoxide hydrolase [6]. Consequently, levels of epoxide hydrolase activity play an important role in determining the fate of PAHs

The highest concentration of the microsomal epoxide hydrolase is in the liver, but it is also encountered in many other tissues including the lung. Despite its high 
level of basal expression, hepatic epoxide hydrolase activity, monitored using benzo[a]pyrene 4,5-oxide as substrate, in the male rat may be induced by a number of xenobiotics, including hepatocarcinogens such as safrole, diethylnitrosamine, aflatoxin $\mathrm{B}_{1}$ and 2-acetylaminofluorene [7,8]. Interestingly, in the same studies no increase was seen following exposure to 3-methylcholanthrene, a PAH that is a potent inducer of CYP1, the principal cytochrome P450 family responsible for PAH metabolism; similar observations have been made in mice [9]. This effect appears not to be tissue specific, as 3-methylcholanthrene also failed to enhance epoxide hydrolase activity in rat lung [10]. By contrast, studies performed using primary human primary hepatocyte cultures demonstrated that a number of xenobiotics could elicit a modest increase in epoxide hydrolase mRNA levels, although this was not translated to the protein level; PAHs were not investigated in this study [11]. In more recent studies emanating from our laboratory employing precision-cut rat liver and lung slices, it was demonstrated that the prototypical PAH benzo[a]pyrene was a modest inducer of epoxide hydrolase activity, and protein and mRNA expression [12].

Clearly, the information available on the modulation of microsomal epoxide hydrolase by PAHs, either in animals or humans, is limited and contradictory despite the pivotal role played by this enzyme in their bioactivation. The objective of the present studies were: (a) to evaluate the ability of six structurally-diverse PAHs to modulate rat epoxide hydrolase activity in precision-cut liver slices; (b) perform similar studies utilising precision-cut human liver slices so as to be able to identify whether a species difference in the regulation of this enzyme exists between human and rat, a very frequently used animal species in the evaluation of the carcinogenic activity of PAHs; (c) establish whether tissue-specific differences are present in the 
regulation of this enzyme by PAHs in the rat liver and lung, the latter being a target tissue of their carcinogenicity and, finally, (d) compare the effects of PAHs on rat hepatic epoxide hydrolase and CYP1A and CYP1B expression determined under identical conditions [13]. Epoxide activity was monitored using one of the primary epoxides of benzo[a]pyrene, namely the 4,5-oxide, as substrate, and was supported by immunological studies employing Western blot analysis; additionally, in order to delineate possible mechanisms, mRNA levels were determined in some of the studies.

\section{Materials and Methods}

Dibenzo[a,1]pyrene, 1-methylphenanthrene (LGC Promochem, Middlesex, UK), benzo[a]pyrene, fluoranthene, benzo[b]fluoranthene, dibenzo[a,h]anthracene, peroxidase-linked anti-rabbit antibody (Sigma Co. Ltd., Poole, Dorset, UK), benzo[a]pyrene 4,5-epoxide and benzo[a]pyrene 4,5-diol (Mid-West Research Institute, Kansas, USA), rat genomic DNA (Novagen, Wisconsin, USA), Superscript II (Invitrogen, Paisley, UK), RNase-free DNase (Promega, Wisconsin, USA), Qiagen RNeasy Mini kits (Crawley, West Sussex, UK), Absolute ${ }^{\mathrm{TM}}$ QPCR Mix (Abgene, Epsom, Surrey, UK) and Earle's balanced salt solution (EBSS), foetal calf serum, gentamycin and RPMI 1640 Glutamax II culture medium (Gibco-BRL Life Technologies, Paisley, Scotland) were all purchased. Twelve-well plates were obtained from Bibby Sterilin (Helena Biosciences, Sunderland). Antibody to microsomal epoxide hydrolase was a kind gift from Professor M Arand [14].

Liver sections from two human cadaveric livers that could not be used for transplantation purposes were obtained from the UK Human Tissue Bank (The Innovation Centre, Oxford Street, Leicester, UK). Donor 1 was a 60-year old male 
and Donor 2 a 57-year old female; both were Caucasian and smokers. Sections were received 8 - 12 hours after the liver was removed from the donor and were transported in cold University of Wisconsin (UW) preservation solution on ice. On receipt, the liver sections were immediately transferred into a sterile container and, after the UW solution was carefully decanted, they were washed 3 to 4 times with culture medium. The metabolic viability of the two livers was evaluated using 7-ethoxycoumarin as substrate, following a 6-hour incubation [13]. Male Wistar albino rats (200g) were obtained from B\&K Universal Ltd (Hull, East Yorkshire, UK). The animals were housed at $22 \pm 2{ }^{\circ} \mathrm{C}, 30-40 \%$ relative humidity in an alternating 12-hr light:dark cycle with light onset at $07.00 \mathrm{hr}$. Rats were killed by cervical dislocation, liver was immediately excised; human and rat liver slices $(250 \mu \mathrm{m})$ were prepared from $8 \mathrm{~mm}$ cylindrical cores using a Krumdieck tissue slicer (Alabama Research and Development Corporation, Munsford, AL, USA) as previously described [15]. The multiwell plate procedure, using tweleve-well culture plates, was used to culture the slices. The culture medium was essentially that described by Lake et al. [16] and comprised RPMI 1640 Glutamax II containing foetal calf serum (5\%), L-methionine (0.5 $\mathrm{mM})$, insulin $(1 \mu \mathrm{M})$, gentamycin $(50 \mu \mathrm{g} / \mathrm{mL})$ and hydrocortisone 21hemisuccinate $(0.1 \mathrm{mM})$. One slice was placed in each well, in $1.5 \mathrm{ml}$ of culture medium. Slices were incubated under sterile conditions on a reciprocating plate shaker housed in a humidified incubator, at a temperature of $37^{\circ} \mathrm{C}$ and under an atmosphere of $95 \%$ air $/ 5 \% \mathrm{CO}_{2}$. The slices were initially pre-incubated for $30 \mathrm{~min}$ in order to slough off any dead cells due to slicing. For the production of lung slices, animals were killed by an overdose of sodium pentobarbital, and lungs were perfused intratracheally with agarose $(0.75 \% \mathrm{v} / \mathrm{w})$ at $37{ }^{\circ} \mathrm{C}$. Agar was allowed to solidify, and lung slices $(600 \mu \mathrm{m})$ were prepared from cylindrical cores $(8 \mathrm{~mm})$ as described for the 
liver [17]. For incubations exceeding $24 \mathrm{~h}$, slices were placed in fresh medium every $24 \mathrm{~h}$. Three different slice pools, comprising 4-10 slices, were used per time point.

Following incubation, slices were removed from the medium, homogenised, and microsomal fractions were prepared. Epoxide hydrolase activity was determined in the microsomal fraction using benzo[a]pyrene 4,5-epoxide as substrate [18]; protein concentration was also determined [19]. Finally, in order to determine expression at the protein level, hepatic microsomal proteins were resolved by electrophoresis and incubated with antibody to epoxide hydrolase, raised in rabbit, and peroxidase-linked anti-rabbit antibody. Immunoblots were quantitated by densitometry using the GeneTool software (Syngene Corporation, Cambridge, UK).

Two slices were pooled for total RNA extraction, and for each sample triplicate extractions were carried out. RNA was extracted using the Qiagen RNeasy Mini kit, and was quantified using a Nanodrop spectrophotometer. Total RNA was treated with RNase-free DNase to remove genomic contamination. Reverse transcription was primed with random hexamers and carried out by Superscript II according to the manufacturer's instructions. To ensure that DNase treated samples were free from genomic contamination, an RT- control was carried out for every RNA sample. cDNA generated from 50ng was amplified using Absolute QPCR Mix with $400 \mathrm{nM}$ primers and $100 \mathrm{nM}$ fluorogenic probe in a total reaction volume of $25 \mu \mathrm{l}$. QPCR reactions were run on an ABI7000 SDS instrument and quantitation was carried out using the $\mathrm{ABI}$ proprietary software against a standard curve generated from rat genomic DNA.

For the quantitative reverse transcription-polymerase chain reaction, the primer and TAMRA/FAM dual labelled probe [12] were designed using the Primer Express software (Applied Biosystems, California, USA) and purchased from MWG, 
Ebersberg, Germany. Each primer and probe set was designed to amplify sequences within a single exon, so that genomic DNA could be used as a standard. The characteristics of PAH-dependent alteration in transcript levels were calculated using Prism v5 (GraphPad Inc, CA) to fit a one-site binding model (hyperbole) to the observed mRNA levels, generating Imax (maximal fold induction) and $\mathrm{EC}_{50}$ (concentration required to achieve one-half Imax) values.

Statistical evaluation was carried out using the Student's t-test.

\section{Results}

Exposure of rat liver slices to a range of concentrations of the various PAHs resulted in a modest increase in activity only in the case of benzo[a]pyrene and dibenzo[a,h]anthracene, with maxima being achieved at about 5-10 $\mu \mathrm{M}$ (Figure 1). The rise in activity was accompanied by a similar increase in protein levels, detected immunologically, with dibenzo[a,h]anthracene being the more potent of the two (Figure 2). Dibenzo[a,1]pyrene led to a $40 \%$ increase in protein levels, but no significant effect could be detected at the activity level. No increase in protein levels was evident following incubation of the liver slices with benzo[b]fluoranthene, fluoranthene and 1-methylphenanthrene (results not shown). At the mRNA level, however, all compounds elevated epoxide hydrolase mRNA levels (Figure 3). $\mathrm{EC}_{50}$ and Imax values are shown in Table 1.

Basal epoxide hydrolase activity in the two human livers employed in the present study was 3-4 times higher compared with the rat slices. When human liver slices were exposed to the same PAHs, under identical conditions to the rat slices, a similar picture emerged in that benzo[a]pyrene and dibenzo[a,h]anthracene elevated epoxide hydrolase activity; however, in contrast to rat liver, a significant increase in 
activity was also observed following fluoranthene treatment (Figure 4); once again maxima were achieved at a concentration of 5-10 $\mu \mathrm{M}$. The increase in activity was accompanied by a rise in apoprotein levels (Figure 5). Benzo[b]fluoranthene, at only one concentration, gave a rise to a $10 \%$ increase in activity which was statistically significant, but no rise in protein levels was observed at all concentrations studied (Figures 4 and 5).

Basal epoxide hydrolase activity in rat lung slices was much lower than the hepatic activity, by approximately one order of magnitude, and when lung slices were incubated with the various PAHs, under the identical conditions employed in the liver studies, a different induction profile emerged. Benzo[a]pyrene, 1-methylphenathrene and, especially, dibenzo[a,1]pyrene were potent inducers, increasing both activity (Figure 6) and protein levels (Figure 7). Maximal increase in activity was attained at about $1 \mu \mathrm{M}$. In contrast, neither activity nor protein levels were elevated by the treatment with fluoranthene or benzo[b]fluoranthene (Figures 6 and 7). Finally, dibenzo[a,h]anthracene caused a clear increase in apoprotein levels, but the rise in activity was not statistically significant.

\section{Discussion}

The use of precision-cut tissue slices to evaluate the induction potential of chemicals is especially advantageous when studying tissues like the lung, which comprises various cell types characterised with different metabolic activity. Moreover, it allows the study of a range of concentrations that would otherwise require large numbers of animals for in vivo studies. 
In the present study we investigated the ability of six structurally-diverse PAHs to up-regulate epoxide hydrolase activity, a key enzyme in the metabolism of PAHs, as well as other chemical carcinogens. These studies were conducted in rat and human liver, and rat lung slices. The optimal conditions of exposure, e.g. concentration range and incubation time, for both liver and lung slices have already been established [12], and were adopted in the current studies.

Basal activities showed clear species and tissue differences. Firstly, rat lung activity was much lower than in the liver, in agreement with the previous observations [20]. Secondly, although based on only two human livers, it appears that epoxide hydrolase activity in rat is about one-half of that observed in humans. This finding contradicts previous studies where no significant difference in activity, determined using the same substrate, was noted; however, the Sprague-Dawley rat was used in these studies [20], compared to Wistar in the current study, and strain differences may exist as has already been established for cytochrome P450 activities. It is pertinent to emphasise that both liver donors were smokers, so that epoxide hydrolase activity could have been in an induced state. However, since epoxide hydrolase is only a modestly inducible enzyme, for example in comparison with CYP1, we believe this is unlikely, although the possibility can not be entirely discarded.

Of the six PAHs studied, only benzo[a]pyrene and dibenzo[a,h]anthracene enhanced significantly epoxide hydrolase activity in rat liver, and the parallel rise in apoprotein levels suggests that increased enzyme availability is the underlying mechanism. To a lesser extent, exposure of the slices to dibenzo[a,1]pyrene led to a rise in the protein levels but this was not reflected at the activity level, perhaps due to the higher sensitivity of the immunological procedure. At the mRNA level, all PAHs studied increased epoxide hydrolase, albeit to different extent, implying 
transcriptional activation or mRNA stabilisation as a contributory mechanism. However, the high epoxide hydrolase mRNA levels, as defined by Imax/EC 50 , and protein levels observed with some of the PAHs were not translated to increased activity. Lack of concordance between epoxide hydrolase mRNA and protein levels has already been reported following exposure of human primary hepatocytes to chemical inducers [11]. Furthermore, lack of correlation between mRNA levels and activity has been documented with many enzymes, and it has been proposed that a threshold exists that has to be exceeded in order for mRNA to be translated, so that activation of the gene at the mRNA level does not always lead to higher protein levels [21]. However, the fact the mRNA levels were monitored in the complete slice but activity/protein in the isolated microsomes could have contributed to this lack of correlation as translation products of mRNA could have been missed. Moreover, as mRNA levels were monitored only at a single time point differences in temporal response may be partly responsible. Of interest is also the fact that a number of independent regulatory regions have been identified in the epoxide hydrolase gene [4], and it is conceivable that the various PAHs act at different transcriptional units. Benzo[a]pyrene enhanced epoxide hydrolase activity but it was a weak inducer of epoxide hydrolase mRNA levels. It may be cautiously inferred that transcriptional activation may not be the major mechanism for the induction of epoxide hydrolase, and that (post)transcriptional processes, such as translation efficiency or protein stabilisation, may be largely responsible.

In the 1980 s a number of studies failed to show induction of hepatic epoxide hydrolase activity, either in the rat or mouse, by 3-methylcholanthrene which at the time was the model PAH employed in induction studies. Clearly, what the present studies have shown is that individual PAHs have the potential to induce this enzyme, 
and one can not generalise on the basis of studies with single compounds. It is also pertinent to point out that the rise in activity is very modest compared with the effects of the same compounds on CYP1 activity monitored by the O-deethylation of ethoxyresorufin [13]. Moreover, CYP1 and epoxide hydrolase induction by individual PAHs appears to involve different regulation pathways, CYP1 being regulated by the aryl hydrocarbon receptor. For example, benzo[b]fluoranthene was the most potent inducer of CYP1 activity among the six studied PAHs whereas it failed to influence epoxide hydrolase activity. It is likely that the formation of the dihydrodiol epoxides would be enhanced if epoxide hydrolase was also upregulated, in addition to CYP1, as this would facilitate the conversion of the primary epoxides to the dihydrodiol, the precursor of the dihydrodiol epoxide. Indeed the formation of benzo[a]pyrene dihydrodiol-epoxide-DNA adducts in the presence of 3-methylcholanthrene-induced rat liver microsomes was markedly enhanced when the activation system was supplemented with purified epoxide hydrolase [22]

Human epoxide hydrolase activity was similarly elevated following exposure to the six PAHs, the extent of induction being similar to that observed in the rat. In both species, benzo[a]pyrene and dibenzo[a,h]anthracene increased activity, but fluoranthene also enhanced the activity in the human slices only. As noted in rat liver, the rise in activity was accompanied by a similarly modest rise in protein levels, suggesting that enhanced enzyme synthesis and/or enzyme stabilisation is the underlying mechanism for these effects.

Although epoxide hydrolase activity in the rat lung was lower than in the liver, it was more inducible in both activity and, in particular, protein level. Substantial variation in the epoxide hydrolase activity was evident between lung slices, which most likely reflect the fact that the lung, in contrast to the liver, has a heterogeneous 
cell population, and enzyme activity may not be uniformly distributed in all cell types [4]. Moreover, the profile of induction by the six PAHs in the lung differed to that seen in the liver in that, in addition to benzo[a]pyrene, the pulmonary activity was also enhanced by 1-methylphenanthrene and dibenzo[a,1]pyrene, the latter being the most potent of the six PAHs studied. As noted in the liver, changes in activity were associated with similar changes in immunologically-determined epoxide hydrolase protein levels. Moreover, peak induction in the lung slices by PAHs occurred at concentrations of about $1 \mu \mathrm{M}$, one tenth of those required for maximal induction in the liver. A possible contributory factor to this difference may be, at least in part, the higher rate of metabolic breakdown of PAHs in the liver compared with lung, leading to a rapid decline in its effective concentration; the rate of metabolism of benzo[a]pyrene to phenolic products in liver slices is markedly higher compared with lung slices (unpublished observations). At concentrations higher than $1 \mu \mathrm{M}$, the inductive effect of PAHs dropped to control values; a similar picture was noted when the induction of CYP1 was studied under identical conditions [13]

An interesting observation is that, in the lung, dibenzo[a,1]pyrene, a highly carcinogenic compound more active than benzo[a]pyrene, was the most potent inducer of epoxide hydrolase. In contrast, relative to other PAHs it is a weak inducer of CYP1A in both the lung and liver and does not modulate CYP1B1 expression in the lung [13]. As the addition of purified epoxide hydrolase to hepatic microsomes enhanced the generation of the benzo[a]pyrene dihydrodiol epoxide [22], implying that it may be rate limiting, it is tempting to speculate that the ability of dibenzo[a,1]pyrene to stimulate pulmonary epoxide hydrolase, which is present at lower levels in the lung compared with the liver, may contribute in part to its high carcinogenic activity. It is relevant to point out that following systemic exposure to 
dibenzo[a,1]pyrene, the majority of DNA adducts in the lung were derived from the dihydrodiol-epoxide [23].

In summary, employing precision-cut slices in vitro, we have demonstrated that rat microsomal epoxide hydrolase is an enzyme inducible by PAHs, albeit to a much lesser extent compared with CYP1. No correlation was evident between induction of CYP1 and epoxide hydrolase, implying different regulatory control. Although lung epoxide hydrolase activity in the rat is lower than in the liver, it appears to be more sensitive to induction by PAHs. The induction profile by the six PAHs studied differed in the two tissues, revealing tissue-specific differences. Epoxide hydrolase was also inducible in the human liver, with a similar profile to that in rat liver. To our knowledge this is the first study establishing induction of epoxide hydrolase in human tissue.

\section{Acknowledgement}

The authors acknowledge with thanks funding of this work by the European Union through the AMBIPAH project, and thank Dr M Arand (University of Zurich, Zurich, Switzerland) for the kind gift of antibodies to epoxide hydrolase and the UK Human Tissue Bank (The Innovation Centre, Leicester, UK) for the provision of fresh human liver. 


\section{References}

[1] O. Pelkonen, D.W. Nebert. Metabolism of polycyclic aromatic hydrocarbons: Etiologic role in carcinogenesis, Pharmacol. Rev. 34 (1982) 189-222.

[2] F.P. Guengerich, W.W. Johnson, T. Shimada, Y-F. Ueng, H. Yamazaki H. S. Langouët. Activation and detoxication of aflatoxin $B_{1}$, Mutation Res. 402 (1998) 121128.

[3] F.P. Guengerich. Cytochrome P450 oxidations in the generation of reactive electrophiles: epoxidation and related reactions, Arch. Biochem. Biophys. 409 (2003) $59-71$.

[4] M. Arand, F. Oesch. Mammalian xenobiotic epoxide hydrolases, in: C Ioannides (Ed.), Enzyme systems that metabolise drugs and other xenobiotics, Wiley \& Sons, Chichester, UK, 2002, pp. 459-484.

[5] P.J. Sherratt, J.D. Hayes. Glutathione S-transferases, in: C Ioannides (Ed.), Enzyme systems that metabolise drugs and other xenobiotics, Wiley \& Sons, Chichester, UK, 2002, pp. 319-352.

[6] H.R. Glatt, C.S. Cooper, P.L. Grover, P. Sims, P. Bentley, M. Merdes, F. Waechter, K, Vogel, T.M. Guenthner, F. Oesch. Inactivation of a diol epoxide by dihydrodiol dehydrogenase but not two epoxide hydrolases, Science 215(1982) 15071509.

[7] R.N. Sharma, H.L. Gurtoo, E. Farber, R.K. Murray, R.G. Cameron. Effects of hepatocarcinogens and hepatocarcinogenesis on the activity of rat liver microsomal epoxide hydrolase and observations on the electrophoretic behavior of this enzyme, Cancer Res. 41 (1981) 3311-3319. 
[8] J.G. Dent, M.E. Graichen. Effect of hepatocarcinogens on epoxide hydrolase and other xenobiotic metabolizing enzymes, Carcinogenesis 3 (1982) 733-738.

[9] B.D. Hammock, K. Ota. Differential induction of cytosolic epoxide hydrolase, microsomal epoxide hydrolase, and glutathione $S$-transferase activities, Toxicol. Appl. Pharmacol. 71(1983) 254-265.

[10] P. Uotila, O. Pelkonen, G.M. Cohen. The effects of cigarette smoke on the metabolism of $\left[{ }^{3} \mathrm{H}\right]$-benzo[a]pyrene by rat lung microsomes, Cancer Res. 37 (1977) 2156-2121.

[11] H. Hassett, E.M. Laurenzana, J.D. Sidhu, C.J. Omiecinski. Effects of chemical inducers on human microsomal epoxide transferase in primary hepatocytes cultures, Biochem. Pharmacol. 55 (1998) 1059-1069.

[12] D.S. Pushparajah, M. Umachandran, K.E. Plant, N. Plant, C. Ioannides. Evaluation of the precision-cut liver and lung slice systems for the study of induction of CYP1, epoxide hydrolase and glutathione S-transferase activities, Toxicology, 231 (2007) 68-80.

[13] D.S. Pushparajah, M. Umachandran, T. Nazir, K.E. Plant, N. Plant, D.F.V. Lewis, C. Ioannides. Up-regulation of CYP1 in rat lung and liver, and human liver precision-cut slices by a series of polycyclic aromatic hydrocarbons; association with the Ah locus and importance of molecular size, Tox. In Vitro (2007) In press.

[14] M.E. Herrero, M. Arand, J.G. Hengstler, F. Oesch. Recombinant expression of human microsomal epoxide hydrolase protects V79 Chinese hamster cells from styrene oxide - But not from ethylene oxide-induced DNA strand breaks, Environ. Mol. Mutagen. 30 (1997) 429-439. 
[15] E. Hashemi, M. Dobrota, C. Till, C. Ioannides. Structural and functional integrity of precision-cut liver slices in xenobiotic metabolism: a comparison of the dynamic organ and multiwell plate culture procedures, Xenobiotica 29 (1999) 11-25.

[16] B.G. Lake, J.A. Beamand, A.C. Japenga, A. Renwick, S. Davies, R.J. Price. Induction of cytochome P-450-dependent enzyme activities in cultured rat slices, Food Chem. Toxicol. 31 (1993) 377-386.

[17] M. Umachandran, J. Howarth, C. Ioannides. Metabolic and structural viability of precision-cut rat lung slices in culture, Xenobiotica 34 (2004) 771-780.

[18] P.M. Dansette, G.C. DuBois, D.M. Jerina. Continuous fluorometric assay of epoxide hydratase activity, Anal. Biochem. 97(1979) 340-345.

[19] M. Bradford. A rapid and sensitive method for the quantitation of microgram quantities of protein utilizing the principle of protein-dye binding, Anal. Biochem. 72 (1976) $248-254$.

[20] J. Lorenz, H.R. Glatt, R. Fleischmann, R. Ferlinz, F. Oesch. Drug metabolism in man and its relationship to that in three rodent species: monooxygenase, epoxide hydrolase, and glutathione S-transferase activities in subcellular fractions of lung and liver, Biochem. Med. 32, (1984) 43-56.

[21] D. Greenbaum, C. Colangelo, K. Williams, M. Gerstein. Comparing protein abundance and mRNA expression levels on a genomic scale, Genom. Biol. 4 (2003) 117-123.

[22] T.M. Guenthner, F. Oesch. The effects of modulation of microsomal epoxide hydrolase activity on microsome-catalyzed activation of benzo[a]pyrene and its covalent binding to DNA, Cancer Lett. 11 (1981) 175-183. 
[23] B. Madahevan, A. Luch, C.F Bravo, J. Atkin, L.P. Steppan, C. Pereira, N.I. Kerkvliet, W.M. Baird. Dibenzo[a,1]pyrene induced DNA adduct formation in lung tissues in vivo, Cancer Lett. 227 (2005) 25-32. 
Table 1: Parameters defining the PAH-dependent effect on epoxide hydrolase mRNA levels

Parameters were calculated using Prism v5 (GraphPad Inc, CA) to fit a one-site binding model (hyperbole) to the observed mRNA levels.

\begin{tabular}{cccc}
\hline PAH & EC $_{\mathbf{5 0}}$ & Imax & Imax/EC $\mathbf{5 0}$ \\
\hline Benzo[a]pyrene & 1.2 & 4.8 & 4.0 \\
Dibenzo[a,h]anthracene & 0.16 & 6.0 & 37.5 \\
Fluoranthene & 0.59 & 11.0 & 18.6 \\
Benzo[b]fluoranthene & 0.21 & 6.3 & 30.0 \\
Dibenzo[a,1]pyrene & 0.63 & 8.5 & 13.5 \\
1-methylphenanthrene & 5.1 & 7.7 & 1.51 \\
\hline
\end{tabular}

Imax, maximal fold induction; $\mathrm{EC}_{50}$, concentration required to achieve one-half Imax. 


\section{Legends to figures}

Figure 1: Concentration-dependent induction of epoxide hydrolase activity by PAHs in precision-cut rat liver slices.

Precision-cut rat liver slices were incubated in the absence and presence of the various PAHs, dissolved in DMSO, at a range of concentrations $(0-100 \mu \mathrm{M})$ for 24 hours. At the end of the incubation period, slices were removed from the media, microsomes prepared and epoxide hydrolase activity determined. Results are expressed as mean \pm SD of triplicate pools of slices.

$* \mathrm{P}<0.05$; ** $\mathrm{P}<0.01$; *** $\mathrm{P}<0.001$

Benzo[a]pyrene data from Pushparajah et al (2007)

Figure 2: Concentration-dependent induction of epoxide hydrolase protein levels by PAHs in precision-cut rat liver slices

Precision-cut rat liver slices were incubated in the absence and presence of PAHs (0$100 \mu \mathrm{M}$ ) for 24 hours. At the end of this period, slices were removed from the media and pooled, solubilised microsomal proteins were prepared and resolved by SDSPAGE before being transferred electrophoretically to nitrocellulose paper. Immunoblot was carried out using anti-rat epoxide hydrolase followed by peroxidaselabelled anti-rabbit IgG; all lanes were loaded with $5 \mu \mathrm{g}$ of protein. Figures above the immunoblot represent percentage values compared with control (DMSO-treated) slices which were set at $100 \%$.

Benzo[a]pyrene data from Pushparajah et al (2007)

Figure 3: Concentration-dependent changes in epoxide hydrolase mRNA levels in precision-cut rat liver slices incubated with PAHs.

Precision-cut rat liver slices were incubated in the absence and presence of the various PAHs, dissolved in DMSO, at a range of concentrations for 24 hours. At the end of the incubation period, slices were removed from the media and total RNA extracted. Epoxide hydrolase mRNA levels were determined by quantitative RT-PCR (TaqMan). Changes are shown as fold-increases compared to control slices incubated with DMSO only. Results are expressed as mean \pm SD where $n=4$.

$\mathrm{P}<0.05 ; * * \mathrm{P}<0.01$

Benzo[a]pyrene data from Pushparajah et al (2007)

Figure 4: Concentration-dependent induction of epoxide hydrolase activity by PAHs in precision-cut human liver slices.

Precision-cut human liver slices were incubated in the absence and presence of the various PAHs, dissolved in DMSO, at a range of concentrations $(0-50 \mu \mathrm{M})$ for 24 hours. At the end of the incubation period, slices were removed from the media, microsomes prepared and epoxide hydrolase activity determined. Results are expressed as mean $\pm \mathrm{SD}$ of triplicate pools of slices.

$* \mathrm{P}<0.05 ; * * \mathrm{P}<0.01 ; * * * \mathrm{P}<0.001$ 
Figure 5: Concentration-dependent induction of epoxide hydrolase protein levels by PAHs in precision-cut human liver slices

Precision-cut human liver slices were incubated in the absence and presence of PAHs $(0-50 \mu \mathrm{M})$ for 24 hours. At the end of this period, slices were removed from the media and pooled, solubilised microsomal proteins were prepared and resolved by SDSPAGE before being transferred electrophoretically to nitrocellulose paper. Immunoblot was carried out using anti-rat epoxide hydrolase followed by peroxidaselabelled anti-rabbit IgG; all lanes were loaded with $5 \mu \mathrm{g}$ of protein. Figures above the immunoblot represent percentage values compared with control (DMSO-treated) slices which were set at $100 \%$.

Figure 6: Concentration-dependent induction of epoxide hydrolase activity by PAHs in precision-cut rat lung slices.

Precision-cut rat lung slices were incubated in the absence and presence of the various PAHs, dissolved in DMSO, at a range of concentrations (0-5 $\mu \mathrm{M})$ for 24 hours. At the end of the incubation period, slices were removed from the media, microsomes prepared and epoxide hydrolase activity determined. Results are expressed as mean \pm SD of triplicate pools of slices.

* $\mathrm{P}<0.05 ; * * \mathrm{P}<0.01$

Benzo[a]pyrene data from Pushparajah et al (2007)

Figure 7: Concentration-dependent induction of epoxide hydrolase protein levels by PAHs in precision-cut rat lung slices

Precision-cut rat lung slices were incubated in the absence and presence of PAHs (0-5 $\mu \mathrm{M})$ for 24 hours. At the end of this period, slices were removed from the media and pooled, solubilised microsomal proteins were prepared and resolved by SDS-PAGE before being transferred electrophoretically to nitrocellulose paper. Immunoblot was carried out using anti-rat epoxide hydrolase followed by peroxidase-labelled antirabbit IgG. Figures above the immunoblot represent percentage values compared with control (DMSO-treated) slices which were set at 100\%. With the exception of fluoranthene and benzo[b]fluoranthene where the same amount of protein was applied in all wells, with the other PAHs twice the amount of protein was added in the case of the control animals; a correction has already been applied in the quantitation data.

Benzo[a]pyrene data from Pushparajah et al (2007) 
Figure 1
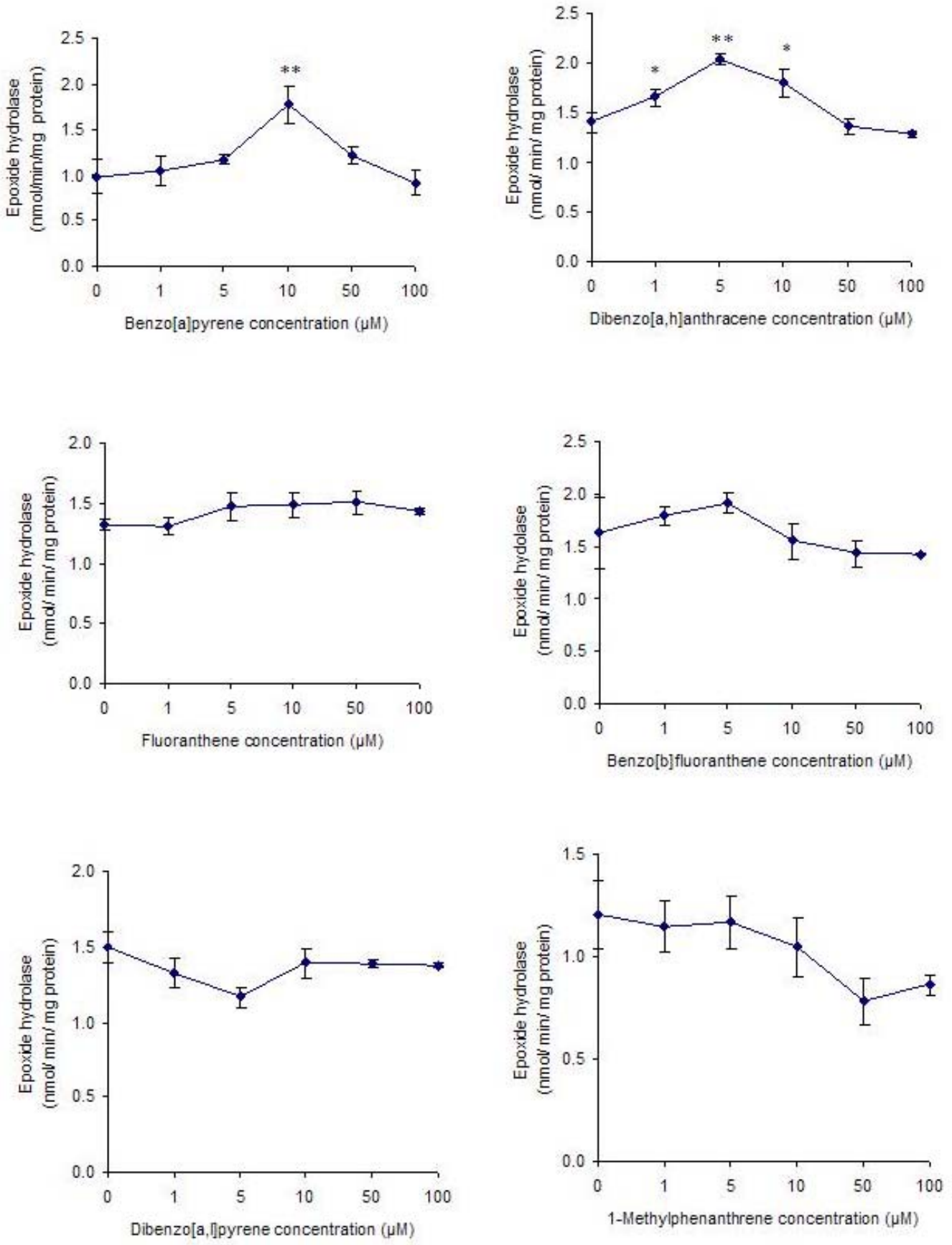
Figure 2
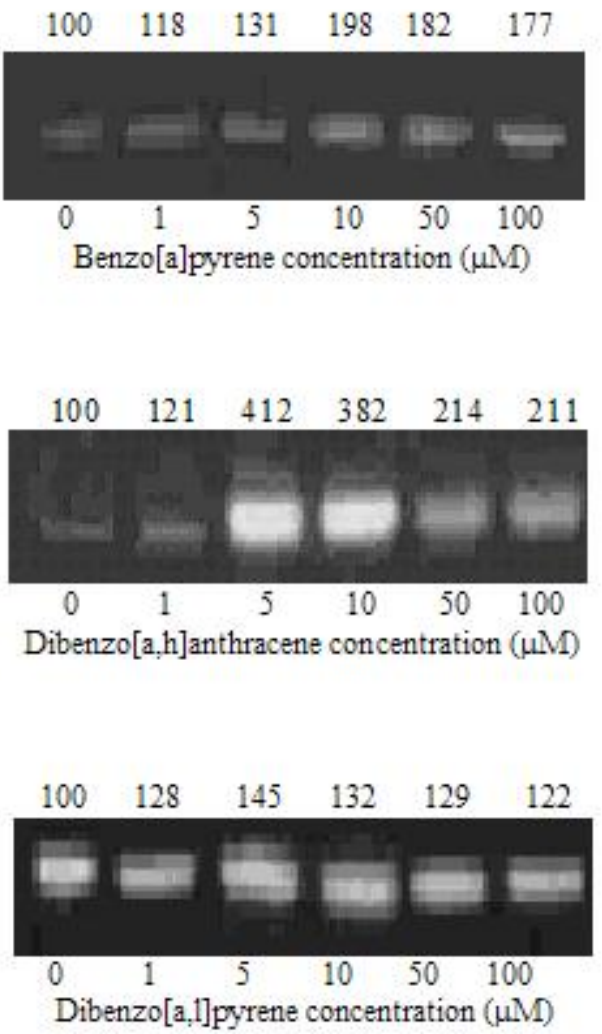
Figure 3
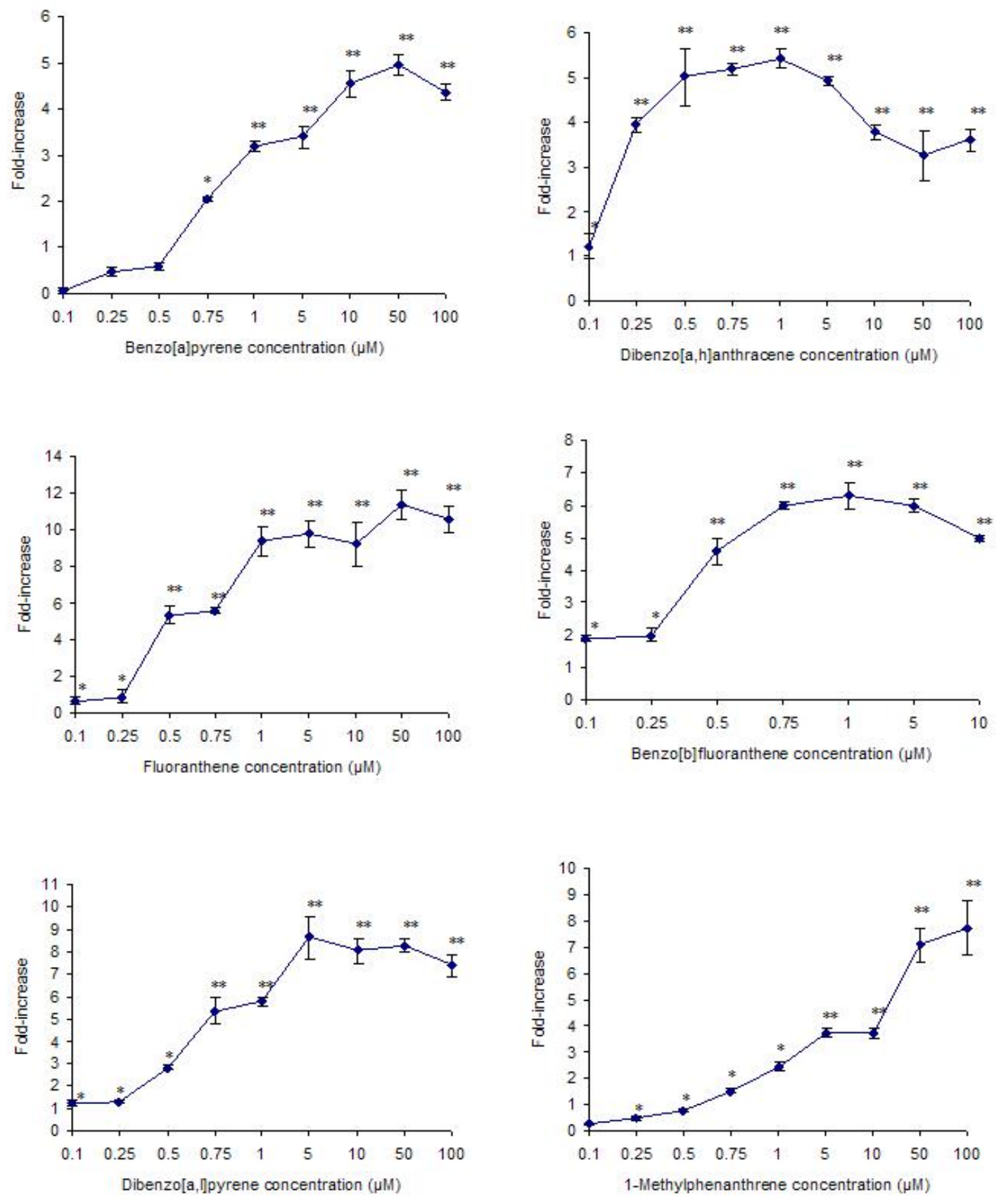
Figure 4
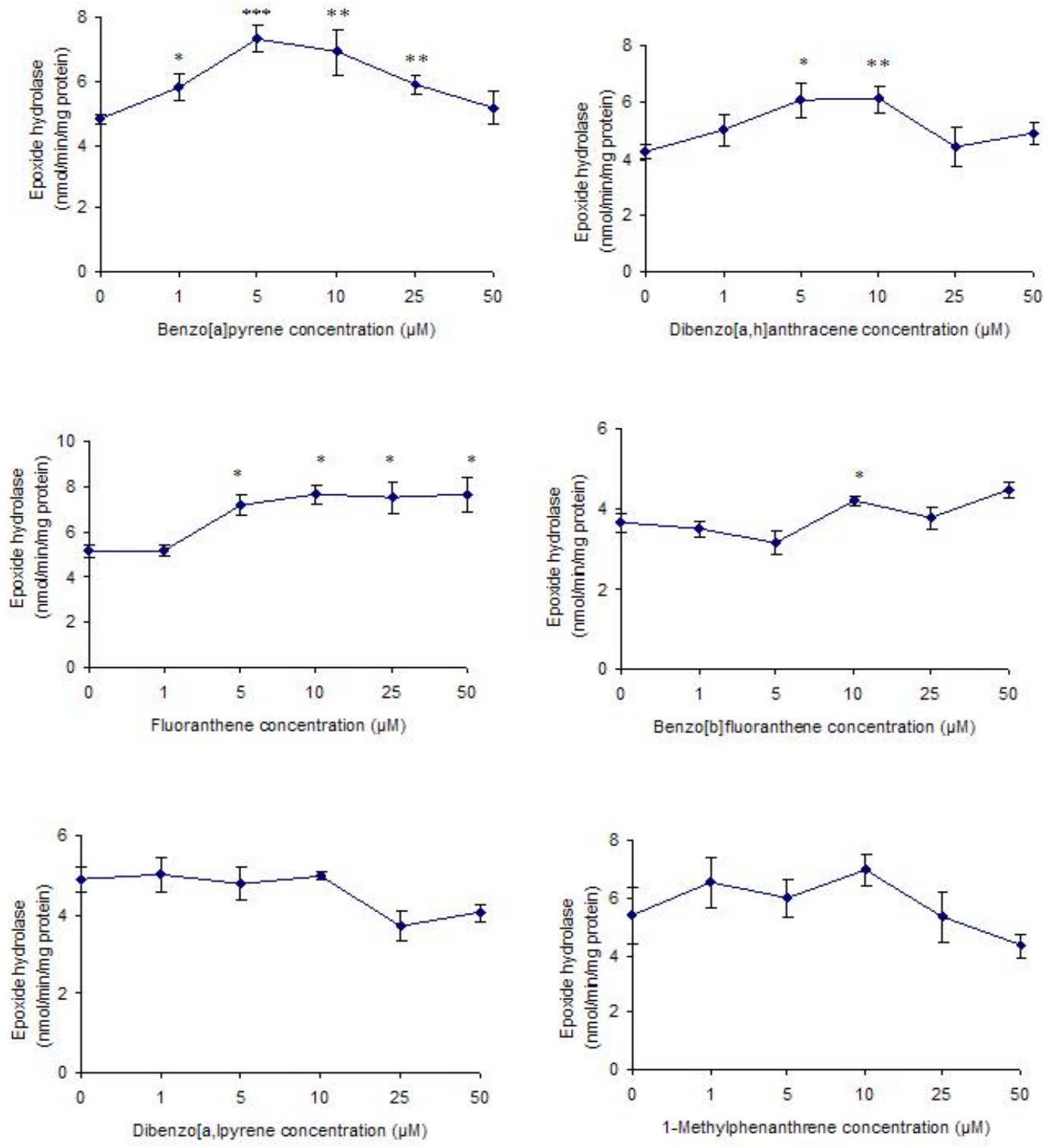
Figure 5
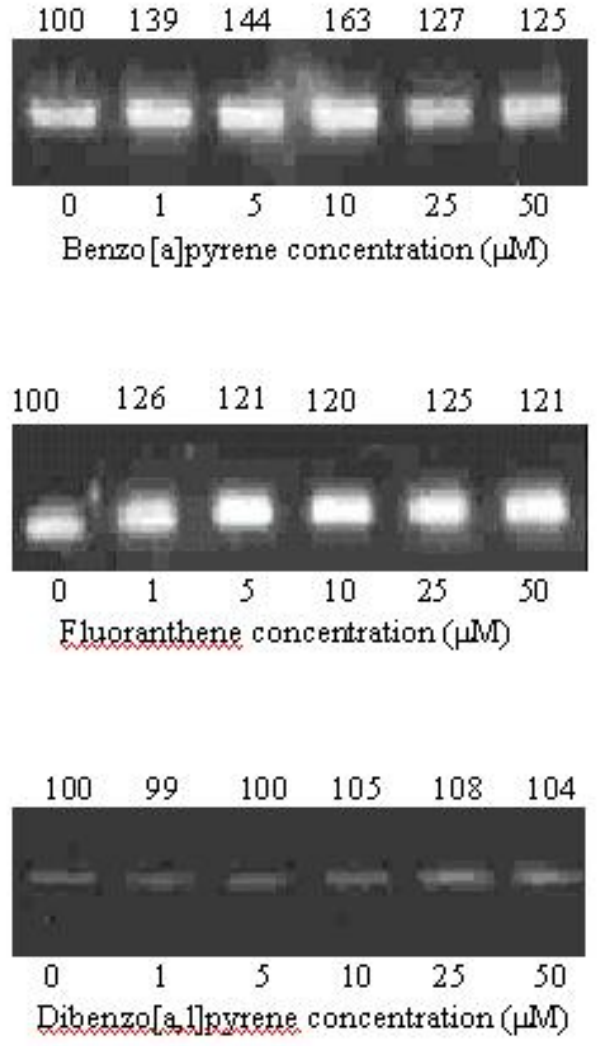

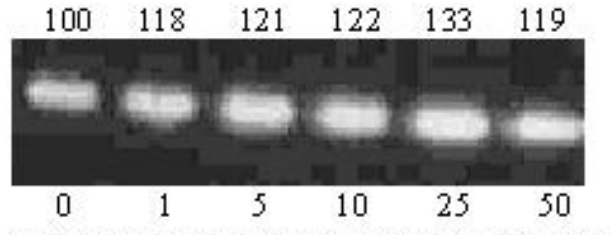

Dibenzo[a,h]anthracene concentration $(\mu \mathrm{M})$
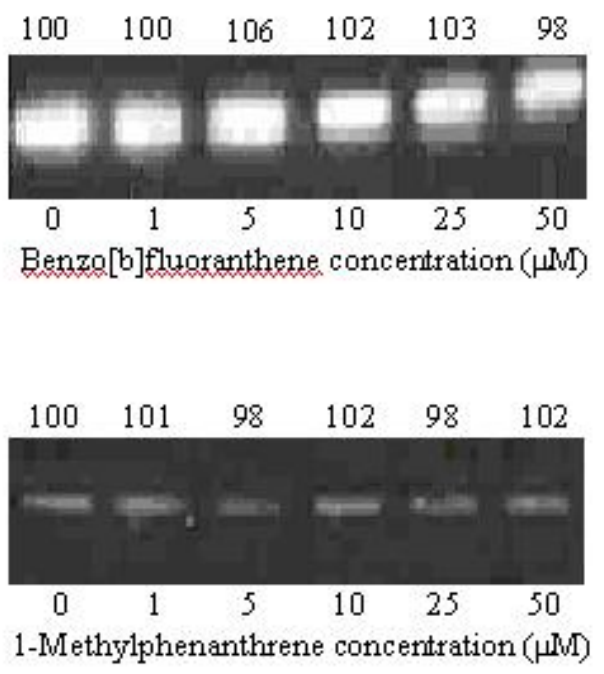
Figure 6
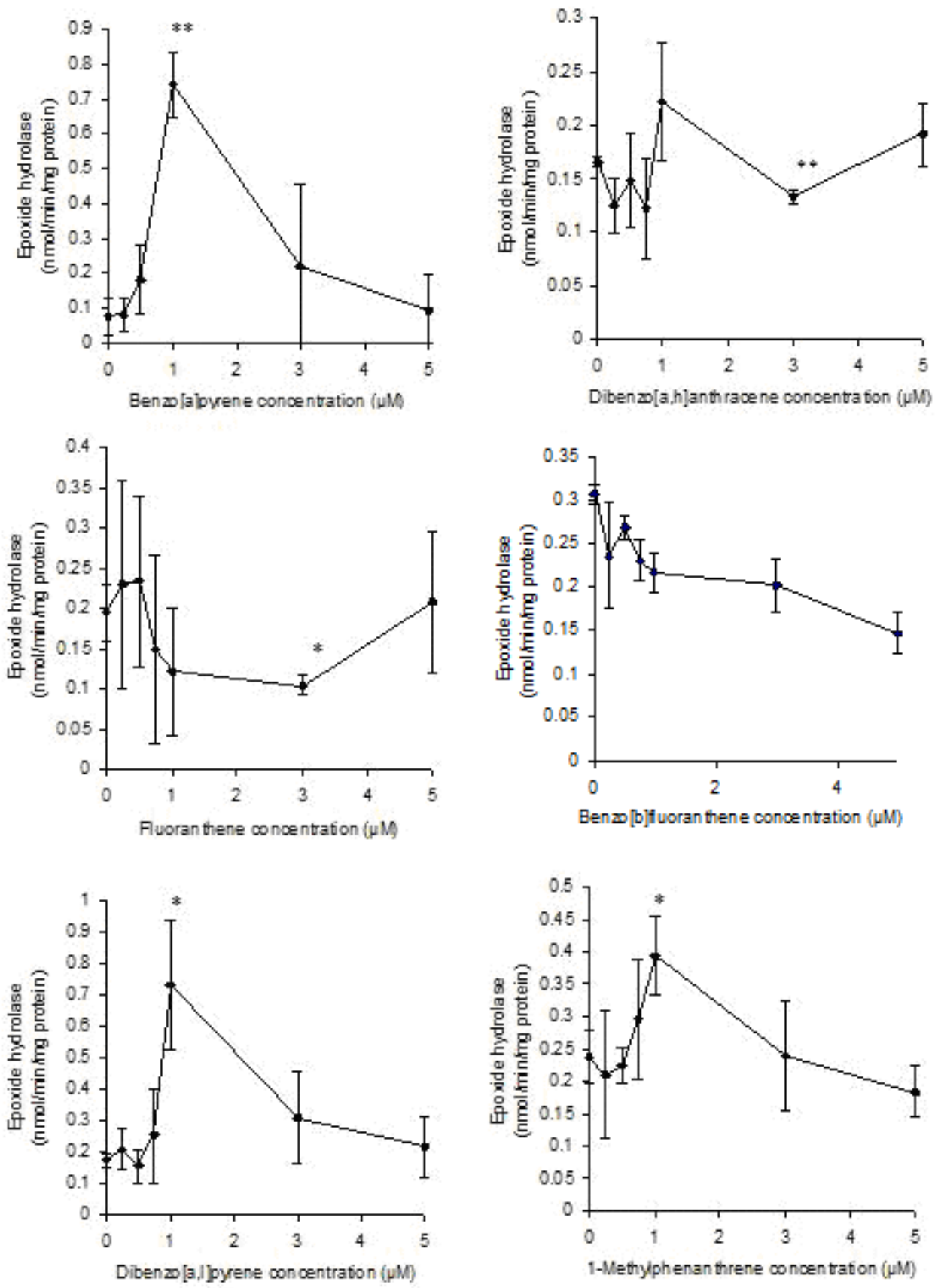
Figure 7

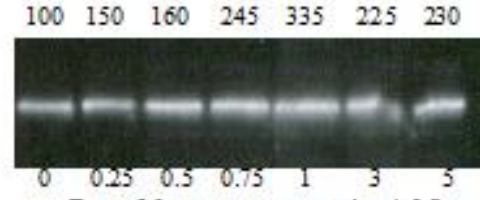

Benzo[a]pyrene concentration ( $\mu M)^{2}$

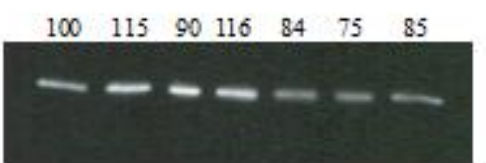

$\begin{array}{lllllll}0 & 0.25 & 0.5 & 0.75 & 1 & 3 & 5\end{array}$ Fluoran thene concentration ( $\mu \mathrm{M})$

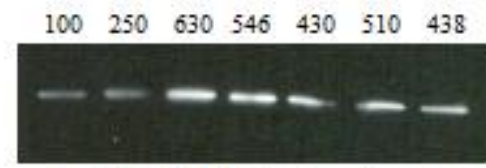

$\begin{array}{lllllll}0 & 0.25 & 0.5 & 0.75 & 1 & 3 & 5\end{array}$

Dibenzo[ $[a, 1]$ pyrene concen tration $(\mu M)$ $\begin{array}{lllllll}100 & 160 & 275 & 220 & 280 & 160 & 150\end{array}$

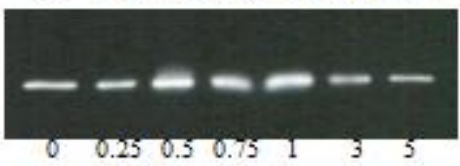

Dibenzo[ a h ] anthracene concentration $(\mu \mathrm{M})$

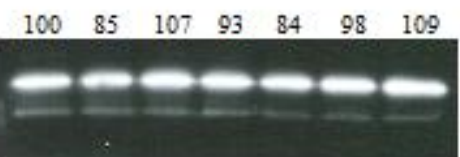

$\begin{array}{lllllll}0 & 0.25 & 0.5 & 0.75 & 1 & 3 & 5\end{array}$ Benzo[b]f hor anthene concentration ( $\mu \mathrm{M}$ )

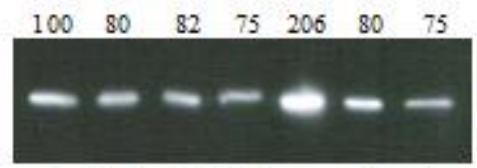

$\begin{array}{lllllll}0 & 0.25 & 0.5 & 0.75 & 1 & 3 & 5\end{array}$

1-Methylphenanthrene concentration ( $\mu M)$ 